\title{
Tangence
}

\section{Régionalismes au Québec au début du siècle}

\section{Annette Hayward}

Numéro 40, mai 1993

Régionalismes littéraires de la francophonie

URI : https://id.erudit.org/iderudit/025763ar

DOI : https://doi.org/10.7202/025763ar

Aller au sommaire du numéro

Éditeur(s)

Tangence

ISSN

0226-9554 (imprimé)

1710-0305 (numérique)

Découvrir la revue

Citer cet article

Hayward, A. (1993). Régionalismes au Québec au début du siècle. Tangence, (40), 7-27. https://doi.org/10.7202/025763ar d'utilisation que vous pouvez consulter en ligne.

https://apropos.erudit.org/fr/usagers/politique-dutilisation/ 


\section{Régionalismes au Québec au début du siècle}

\section{Annette Hayward *}

Comme le laisse entendre notre titre, il est presque impossible d'arriver à une définition univoque de ce que l'on entend par * régionalisme * au Québec au début du siècle. L'implantation du mouvement a beau donner lieu à une querelle entre les régionalistes et les "exotiques" qui finira par polariser toute la scène littéraire vers $1918-1920$, le sens que l'on prête à ce terme varie énormément selon l'étape de la querelle dont il est question et souvent même selon chaque participant.

\section{La Société du Parler français: berceau du mouvement régionaliste canadien}

C'est à Adjutor Rivard, semble-t-il, que doit revenir le mérite d'avoir lancé un mouvement régionaliste au Canada français ${ }^{1}$. En tant que principal critique littéraire du Bulletin du Parler français au Canada (1902-1918) ${ }^{2}$, c'est lui surtout qui s'est ouvertement chargé de promouvoir auprès des écrivains canadiens l'émulation du mouvement régionaliste français:

Nous avons imité la France, même quand elle ne faisait qu'enfiler des coquillages sonores, et nous avions vraiment trop l'air de dire, avec le poète mis à la scène par Coppée:

"Qui pourrais-je imiter, pour être original?.

Imitons-la aujourd'hui - pour mieux dire, imitons ses poètes régionalistes, ses poètes du terroir, qui se penchent vers la glèbe et chantent la bonne terre, avec un élan vers Celui qui

*Fait, sous le clair soleil, croître l'herbe des champs *.

- Annette Hayward est professeure à l'Université Queen's à Kingston. Elle a publié plusieurs articles et rédigé une thèse de doctorat sur le conflit entre les régionalistes et les * exotiques * au Québec (1900-1920).

1 C'est ainsi qu'on identifiait alors la *nation", le * pays*, l'entité dont on défendait la culture, les traditions, la religion et la langue. D'ailleurs, le Canada français pouvait inclure, au besoin, les Franco-Américains. Ce n'est qu'avec Maria Chapdelaine que naîtra l'expression du *pays de Québec.. Le Pays laurentien, quant à lui, avait proposé la * Laurentie....

2 Camille Roy y jouera surtout le rôle d'historien de la littérature canadienne-française. 
Ces poètes de la décentralisation, en France, montrent comment les nôtres pourraient nous créer une autonomie littéraire, leurs vers tendent, suivant une expression heureuse, à "instaurer le culte des petites patries pour fortifier celui de la grande $* 3$.

Pour avoir une poésie à nous, et qui incarne l'âme francocanadienne, chantons donc avant tout, notre "petite patrie *4.

Rivard a commencé sa campagne, en octobre 1903, en inaugurant dans le Bulletin du Parler français au Canada (une revue essentiellement linguistique ${ }^{5}$ ) une série d'articles destinés à faire connaître au Québec les poètes régionalistes de la France ${ }^{6}$. Par la suite, il publiera quarante-quatre comptes rendus élogieux d'ouvrages liés à ce mouvement et signalera aux lecteurs de la revue les événements importants qui marquent son évolution. Le régionalisme français devient ainsi une de ses spécialités; lorsqu'un comité d'étude de la Société du Parler français se forme à Montréal en juin 1910, Rivard prononce pendant la cérémonie d'inauguration une conférence sur les poètes régionalistes.

Il faudra beaucoup de temps, cependant, avant que le message régionaliste ne se répande à travers toute la province. Le texte cité ci-dessus date de 1909, ce qui montre que, pour Rivard,

3 Il est clair que le régionalisme ici ne vise nullement à contester ou remplacer le centre, mais seulement à en partager le pouvoir.

4 Adjutor Rivard, "Les livres. Albert Ferland, Le Canada cbanté., Bulletin du Parler français (dorénavant PF) vol. VIII, n' 2, octobre 1909, p. 75.

5 "Organe de la "Société du Parler français au Canada", le Bulletin est consacré à l'étude de notre langue. Son programme est celui de la Société: la PHONETIQUE, - le LEXIQUE, - la MORPHOLOGIE, - la SYNTAXE du langage populaire. Ajoutons: "l'étymologie populaire", la "dialectologie composée", la "terminologie technique", la "littérature orale $^{n}$, et en général les considérations philologiques, ethnologiques et littéraires qui peuvent aider au développement, à la conservation, au perfectionnement de la langue française au Canada et dans les centres canadiens de la Nouvelle-Angleterre., (Le Comité du Bulletin, *Aux lecteurs, $P F$, vol. I, n' 1 , septembre 1902 , p. 1.

6 Ces articles, parus entre octobre 1903 et décembre 1904, portent sur Gabriel Nigond (du Berry), les "Vers saintongeois*, Louis Beuve (de Normandie), Anatole Le Braz (de Bretagne), Charles Lamy (poète flamand), Achille Millien (du Nivernais), Paul Harel (de Normandie), l'abbe Justin Bessou (poète rouergat), Ernest Millet (de Normandie) et Louis Tiercelin (de Bretagne) et puisent souvent leurs renseignements dans l'anthologie régionaliste d'Albert Grimaud parue en 1903. Deux autres études, sur Joseph-Emile Poirier, de Bretagne, et Georges Gourdon, de Saintonge, paraitront en 1909. 
la bataille n'était pas encore gagnée?. Vers 1912-1913, en revanche, le régionalisme exerce un ascendant de plus en plus grand, à Montréal comme à Québec. En 1916, certains commencent à parler du "triomphe " de l'école régionaliste au Québec, et en 1918 les «autres " (les "exotiques") se sentiront obligés de se regrouper autour du Nigog afin de faire entendre un autre son de cloche.

L'initiative de Rivard n'aurait pas connu autant de succès, cependant, sans le concours du programme de "nationalisation de la littérature canadienne * lancé par l'abbé Camille Roy, au nom de la Société du Parler français, dans son célèbre discours de décembre 1904. Nouvellement revenu de la Sorbonne et bien décidé à prouver l'existence de la littérature canadienne-française afin de pouvoir se poser en digne émule de son ancien professeur, Émile Faguet, et de son maître à penser, Ferdinand Brunetière, Camille Roy avait déjà commencé dans le Bulletin une série d'articles qui allaient être à l'origine de la première (et, pendant longtemps, la seule) histoire de la littérature canadienne-française. Il faut remarquer cependant qu'il était uniquement question, dans ce discours, de "nationalisation de la littérature canadienne ", jamais de "régionalismex. L'influence la plus nette qui s'en dégage est celle de l'abbé Henri-Raymond Casgrain. Mais si les propos du futur doyen des lettres canadiennes veulent perpétuer l'esprit catholique, nationaliste et conservateur de l'École patriotique de 1860 , ils visent aussi (et peut-être surtout) à contrer l'influence trop moderniste de certains écrivains de l'École littéraire de Montréal, en particulier celle du "pauvre "Émile Nelligan dont on vient de publier un recueil de poésies: "Il ne faut pas égarer sur des sujets étrangers, ou gâter par des procédés exotiques notre littérature canadienne .8 . Voilà le genre de phrase que le comité de la revue, et surtout Adjutor Rivard, se feront un plaisir de répéter.

Au modèle suspect de Nelligan, les rédacteurs du Parler français préfèrent nettement celui des Gouttelettes de Pamphile

7 L'École littéraire de Montréal venait pourtant de reconnaître leur *devoir patriotique. (C. Gill) et de fonder une revue intitulée Le verroir. Et Albert Ferland n'a certainement pas besoin d'être converti au sujet canadien du terroir. Il faut croire que l'absence d'une allégeance spécifique au * régionalisme = et la tolérance envers un certain éclectisme qui persistait quand même au sein de cette École classaient encore ses membres, pour Rivard, parmi les gens à convertir.

8 Camille Roy, · La nationalisation de la littérature canadienne , $P F$, vol. III, $\mathrm{n}^{\circ}$ 4, décembre 1904, p. 117. 
Lemay, dont certains morceaux auraient pu figurer avec honneur, nous dit-on, dans La race et le terroir, une anthologie de poésie régionaliste de France: «Depuis [la lecture de cette anthologie], une pensée m'obsède et c'est que la province de Québec, étant elle aussi province de France, ne soit pas représentée dans le beau livre de M. Albert Grimaud "9.

Rivard, en tant que principal critique littéraire du Bulletin, affirme procéder essentiellement comme Camille Roy le faisait dans ses volumes de critique, c'est-à-dire en signalant d'abord les qualités de l'ouvrage, afin d'encourager l'auteur, et en parlant seulement vers la fin des éléments qu'on pourrait améliorer. Il avoue cependant ne pas toujours y réussir, étant uforcé de parler aussi de livres qu'il est impossible de traiter avec bienveillance " ${ }^{10}$. En réalité, Rivard est porté à condamner beaucoup plus catégoriquement que son confrère les tendances littéraires modernes, que ce soit le réalisme, le symbolisme ou d'autres écoles expérimentales de l'époque ${ }^{11}$. Sa critique se distingue aussi par une tendance à intégrer à sa critique des commentaires étrangers à l'œuvre et destinés à encourager les écrivains dans la direction d'une littérature régionaliste.

En effet, si l'abbé Camille Roy appuie fortement l'emploi du sujet canadien, il évite longtemps d'utiliser le mot "régionalisme" et parle beaucoup plus volontiers du "sol natal " et des "choses canadiennes". Pourtant, il ne s'oppose pas à ce terme lorsque le comité de la revue, et en particulier Rivard, interprètent son "programme" comme un plaidoyer en faveur d'un régionalisme littéraire canadien. Cela correspond bien à la politique générale de la revue, d'ailleurs, qui donne toujours l'impression d'une unanimité parfaite. De plus, les écrits de Camille Roy affectionnent la méthode de la douce persuasion et préfèrent louvoyer ou se contredire plutôt que de

9 J.-E. Prince, «Les Gouttelettes... *, PF, vol. II, no 10, été 1904, p. 315. Nous soulignons. Avec cet article, Prince devient, à l'été 1904, le premier collaborateur de la revue à annoncer sans équivoque que la source de l'autonomie littéraire du Québec se trouve dans les œuvres du terroir et dans l'imitation des régionalistes français. Le travail d'initiation de Rivard a de toute évidence porté fruit.

10 Adjutor Rivard, - Livres et revues. L'abbé Camille Roy, Essais sur la littérature canadienne., PF, vol. VI, n' 1 , septembre 1908, p. 22.

11 Cf. PF, vol. VII, $\mathrm{n}^{\circ} 10$, été 1909, p. 379-380. Camille Roy, lui, condamne rarement de façon catégorique et se contente, par exemple, de conseiller un réalisme * idéaliste ", c'est-à-dire des descriptions précises et détaillées qui chantent les sélérnents sains et vrais . 
contredire ouvertement autrui ${ }^{12}$. Il est donc fort probable que Camille Roy n'ait jamais partagé entièrement l'engouement de son collègue pour le mouvement régionaliste, ce qui aiderait à expliquer certaines réticences qu'il exprime à ce sujet vers l'époque où il accepte finalement d'associer comme synonymes le terroir et le régionalisme. Ainsi, après avoir écrit à l'été 1917 que "Par nos champs et nos rives est l'un des meilleurs recueils que nous ayons de poésie régionaliste, de poésie du terroir" $\left(P F\right.$, vol. $\mathrm{XV}, \mathrm{n}^{\circ} 10$, p. 460), il précise quelques mois plus tard qu'une poésie du terroir

[...] n'est ni la plus large, ni la plus puissante, ni la plus divine. [...] Mais il faut que toutes les poésies de la nature et de la vie trouvent leur expression humaine, et c'est un grand mérite pour le poète que de savoir reconnaître son domaine, sa province intellectuelle, le lieu natal de ses rêves et de ses inspirations (PF, vol. XVI, n 5, p. 194-195).

Plus d'un an auparavant, d'ailleurs, il avait critiqué (avec douceur) ceux qui voyaient dans les tableautins rustiques des Rapaillages "la voie * de la littérature canadienne, en précisant qu'une telle voie n'était pas vraiment nouvelle. Il prend même la peine d'ajouter que les auteurs des Rapaillages, du Canadien errant ou de Chez nous "se sont peut-être avisés de marcher sur "l'aubel du chemin"; peut-être ont-ils même exploré un petit sentier parallèle, déjà tracé aussi, et où il y avait à cueillir encore quelques fleurs "13. La même année (1916), pourtant, Roy avait réaffirmé ailleurs sa conviction en la nécessité d'ancrer la littérature canadienne dans *le culte de Dieu et l'attachement au sol *: "c'est en ce sens qu'il convient d'orienter toujours, et de mieux en mieux, notre activité littéraire; c'est à cette condition principale que notre littérature se pourra distinguer de toutes les autres littératures de langue française. ${ }^{14}$. Il semblerait donc que Camille Roy, tout en croyant fermement qu'une littérature canadienne nécessite l'emploi du sujet canadien - et pas n'importe quel sujet canadien (prêtrise oblige?) -, hésite cependant à la

12 Dans son discours sur \& La nationalisation de la littérature canadienne *, par exemple, il conseille aux écrivains d'écrire sur n'importe quel sujet, à condition que ce soit un sujet canadien.

13 Camille Roy, «Les livres: L'abbé Lionel Groulx. Les Rapaillages ", PF, vol. XV, $n^{\circ} 4$, décembre 1916, p. 170. Ce n'est sans doute pas un hasard si ces premières réserves explicites ont été provoquées par le succès du recueil de Lionel Groulx, vu l'écart idéologique qui sépare ces deux hommes.

14 Camille Roy, "Préface ", dans La croix du chemin. Premier concours littéraire de la Société Saint-Jean-Baptiste de Montréal, Montréal, s.é., 1916, p. 10-11. 
restreindre à l'inspiration régionaliste tant prônée par Rivard et d'autres confrères de la revue.

Au Bulletin du Parler français, en effet, les termes "régionaliste " et "terroir " s'utilisent souvent comme de simples synonymes de "Canadien"15. Le Comité du Bulletin ira jusqu'à dire: "Régionalisme, décentralisation, nationalisme, ces mots sont synonymes " ( $P F$, vol. III, n 6, p. 185). À vrai dire, les collaborateurs de la revue, malgré leurs prétentions philologiques, se servent de toute cette terminologie un peu au hasard, dans la mesure où elle peut leur être utile. Ils ont certainement tendance à accoupler sans distinction les mots "régionaliste " et "terroir ". Quant à la confusion entre "canadien " et "régionaliste", elle se comprend facilement si l'on se rappelle que plusieurs de ces critiques aiment considérer le Québec comme une province de France; la littérature produite ici devient alors automatiquement une littérature régionale ou plutôt, vu sa volonté de se distinguer de la production parisienne de l'époque, régionaliste. Mais qui dit "littérature régionaliste" à cette époque dit aussi et avant tout "littérature du terroir", en France comme au Canada, car l'idéologie du mouvement veut que le terroir soit le thème régionaliste par excellence. De plus, l'idéologie agriculturiste qui domine au Québec à cette époque prêche que le seul * vrai * Canadien français exerce le métier de paysan.

Henri d'Arles (l'abbé Henri Beaudé), autre collaborateur du Bulletin, adopte pourtant une perspective différente. S'il confond lui aussi les termes "régionaliste * et "terroir", il ne leur prête pas du tout un sens équivalent à "canadien *. Pour lui, le Québec, comme la France, se compose de plusieurs régions et une littérature régionaliste en ferait ressortir les particularités. Il reproche donc à Blanche Lamontagne de ne pas suffisamment mettre en valeur, dans Visions gaspésiennes, ce qui distingue la Gaspésie de la région montréalaise ou de la côte de Beaupré ( $P F$, vol. XII, n 4, p. 127-128).

\section{Considérations terminologiques}

L'impression d'unanimité qui se dégage du Bulletin du Parler français est donc trompeuse, comme l'est le flou terminologique qui se rencontre tout au long de la querelle des régionalistes et des "exotiques". En général, cependant, le terme régionalisme

15 Rappelons que "canadien - est synonyme de "canadien-français* à cette époque. 
était, pour la plupart de ses partisans, synonyme de "littérature canadienne", cette dernière impliquant à son tour une grande variété de conceptions. Pour les "exotiques", en revanche, le terme "régionaliste" se confond rapidement avec "chauvin, provincial, campagnard, bigot, francophobe, anti-moderniste, séparatiste. D'ailleurs, l'usage inconsidéré de ce mot ne se restreint pas à l'époque de la querelle.

Il peut donc être extrêmement tentant, en retraçant l'histoire littéraire de cette époque, de faire abstraction du mot "régionaliste " et de considérer toutes les variations de ce mouvement comme autant de formes de nationalisme ayant pour but final la formation d'une littérature canadienne-française autonome. "Régionalisme" deviendrait alors un simple synonyme de "nationalisme". Mais comment désigner alors de grands nationalistes comme Olivar Asselin ou Jules Fournier, qui se sont ouvertement inscrits en faux contre le mouvement régionaliste, et contre presque tout ce que celui-ci pouvait représenter ${ }^{16}$ ? À bien y penser, que veut dire exactement le terme "nationalisme"? Est-il simplement synonyme de patriotisme? Être nationaliste dans un État-nation et être nationaliste au Québec, est-ce le même phénomène? Le régionalisme et le nationalisme peuvent-ils vraiment être synonymes?

Une petite incursion du côté des dictionnaires et encyclopédies révèle qu'il s'agit de deux mots relativement récents et dont la définition fluctue beaucoup selon les époques. Le terme "nationalisme " n'apparaît qu'à l'extrême fin du xvII ${ }^{\mathrm{e}}$ siècle et, selon l'Encyclopaedia universalis,

Il n'est guère de mot, dans le vocabulaire historique et politique de l'époque contemporaine, plus chargé d'ambiguïté que celui de nationalisme. [...] il semble cependant qu'une formulation générale puisse être présentée, susceptible de recouvrir les réalités les plus communément reconnues du phénomène nationaliste. Dans la situation antérieure à l'existence d'un Étatnation juridiquement autonome, le nationalisme semble pouvoir être défini comme la volonté d'une collectivité ayant, par suite des circonstances diverses, pris conscience de son individualité historique de créer et de développer son propre État souverain. Dans le cadre d'un État-nation déjà constitué, il peut apparaître,

16 L'action de Jules Fournier est allée jusqu'à publier une série d'articles qui tentaient de prouver que le Canadien français n'avait jamais eu la vocation agricole et qu'il avait toujours accepté de devenir cultivateur faute de mieux... 
sur le plan politique, comme le souci prioritaire de défendre l'indépendance et d'affirmer la grandeur de l'État-nation, l'adjectif prioritaire établissant nettement la différenciation entre patriotisme et nationalisme [...]. ${ }^{17}$

Quant au terme "régionalisme", il date de la seconde moitié du XIX ${ }^{e}$ siècle et ne figure même pas dans le Grand Dictionnaire universel $d u$ XIX siêcle de Pierre Larousse. Le traitement qu'il reçoit de la part des dictionnaires et encyclopédies semble nettement plus mélioratif depuis qu'on l'utilise, en cette deuxième moitié du $\mathrm{xx}^{e}$ siècle, pour désigner une "Doctrine de politique internationale qui consiste à favoriser la création de liens particuliers entre les diverses nations d'un même continent *. Au début du $x x^{e}$ siècle, cependant, ce sont plutôt les autres acceptions du mot qui ont cours:

1. $V x$. Doctrine politique et sociale dont le principe est de favoriser, au sein d'une même nation, des groupements régionaux conformes aux divisions imposées par la géographie et l'histoire. /2. Tendance à conserver et à développer les caractères originaux d'une région. /Spécialem. Caractère d'une œuvre littéraire ou artistique, d'un écrivain ou d'un artiste qui choisit ses sujets, ses personnages dans une région déterminée: $L e$ régionalisme des romans d'Henri Pourrat.

Il n'est peut-être pas non plus inutile d'ajouter le dernier élément de cette définition afin de se rappeler que le régionalisme désigne également, en linguistique, "une locution propre à une région "18. Au Parler français, par exemple, ce sont les préoccupations linguistiques qui ont mené au régionalisme.

Ce qui est certain, c'est que le terme "régionalisme " est indissociable de l'idée d'un centre qui se situe ailleurs. Et si une littérature arégionale " désigne tout simplement une littérature qui

17 Georges Vedel, - Régionalisme *, Encyclopaedia Universalis, tome 15, Paris, Encyclopaedia Universalis, 1985, p. 945-946. La définition donnée pour - nationalisation. est également très intéressante, surtout lorsqu'on pense au lien qui se crée entre le sprogramme de nationalisation proposé par Camille Roy et l'idéologie agriculturiste: "La nationalisation est "l'opération par laquelle la propriété d'une entreprise ou d'un groupe d'entreprises est transférée à la collectivité afin de la soustraire, dans l'intérêt général, à la direction capitaliste" (J. Rivero, Le régime des nationalisations, Jurisclasseur civil, annexes III, 1948). (Encyclopaedia Universalis, p. 947).

18 Grand Larousse de la langue française en sept volumes, tome 6, Paris, Librairie Larousse, 1977 , p. 4997 . Cette définition est la plus neutre trouvée. 
souffre d'une réception problématique à cause de son éloignement des assises les plus importantes de l'institution littéraire centrale, une littérature arégionaliste " implique, quant à elle, une volonté de se distinguer du centre, de constituer elle-même une sorte de centre secondaire avec ses valeurs, son idéologie, parfois son esthétique, peut-être même sa propre langue (comme avec Mistral). La spécificité d'un tel "régionalisme " viendrait d'un sentiment de différence ancrée dans l'histoire, les traditions, les mœurs, bref, une différence culturelle, mais n'impliquerait pas la volonté de se séparer totalement du centre afin de former luimême le «vrai - centre. Le régionalisme ne serait donc pas normalement associé à un "nationalisme" qui aurait pour but final la fondation d'un État-nation totalement séparé du centre actuel.

En ce qui concerne le Québec au début du siècle, on peut y distinguer essentiellement - en simplifiant beaucoup - quatre formes ou conceptions du régionalisme.

\section{Le Parler français: un régionalisme décentralisateur et bon-ententiste}

La première de ces conceptions serait évidemment celle mise de l'avant par la Société du Parler français. Essentiellement conservateur et fort catholique, comme l'était aussi son programme linguistique, ce régionalisme visait une certaine autonomie culturelle et linguistique ${ }^{19}$ face à la France (considérée comme le centre dont le Canada français serait une "province intellectuelle"), mais il n'est jamais question de s'en affranchir totalement. Le fait de conseiller aux écrivains d'émailler leurs textes de locutions populaires canadiennes, par exemple, visait surtout à attirer l'attention et l'intérêt d'un public français. La Société avait d'ailleurs adopté une politique explicite de bon-ententisme ${ }^{20}$ et préférait de toute

19 On proposait aux écrivains d'utiliser des canadianismes clans leurs écrits, et chaque numéro du Parler français donnait une liste de mots qui seront réunis plus tard dans l'important Glossaire du Parler français au Canada.

20. Euvre pacifique, la Société évite toute discussion acrimonieuse et se borne à revendiquer les droits que notre loi reconnaît à la langue française. Sans tenter de proscrire l'usage d'aucun autre idiome, elle veut entretenir chez les Canadiens français le culte de la langue maternelle, les engager à conserver pur de tout alliage, à défendre de toute corruption, le parler de leurs ancêtres. (Le Comité du Bulletin, "La Société du Parler français au Canada *, PF, vol. II, n 1 , septembre 1903, p. 9) 
16

évidence assurer autant que possible la protection du français tout en restant en bons rapports avec ceux qui ne partagaient pas la même langue ou la même idéologie. La littérature étant conçue comme un instrument, soit une arme de plus dans la lutte pour la survie de la langue et de la culture française en Amérique du Nord, elle se devait donc (et le mot "devoir " reviendra souvent) de promouvoir une image idéaliste et attirante de ce passé (traditions, légendes, nature, histoire, religion) auquel il s'agissait de rester fidèle. Dans une telle perspective, le présent (l'industrialisation, le mouvement de migration vers les villes) se trouvait automatiquement condamné. Il était à ce moment-là logique de tourner aussi le dos à la littérature française moderne (et à la France moderne) pour s'ouvrir au mouvement régionaliste qui prônait lui aussi la fidélité aux ancêtres, à la terre, à la race, et par-dessus tout à la religion catholique (cette religion alors si menacée par le combisme du gouvernement français). Le rêgionalisme du Parler français n'était donc pas vraiment * nationaliste. dans le plein sens du terme, si l'on accepte que le nationalisme implique une volonté d'indépendance face au centre.

\section{Le régionalisme séparatiste de L'action française}

On ne pourra pas dire la même chose, cependant, de cette deuxième forme de régionalisme qu'est celui préconisé par L'action française sous l'égide de l'abbé Lionel Groulx. Si c'est avec ce grand historien et ses Rapaillages, comme le laisse entendre Jean-Charles Harvey, que le régionalisme littéraire prend véritablement son essor au Québec, il s'agit d'un régionalisme très différent. Au désir d'une autonomie culturelle s'ajoute maintenant une certaine gallophobie, comme diraient ses adversaires, c'est-à-dire une volonté de se débarrasser de l'ombre écrasante de la France; Groulx est peut-être un des premiers à avoir parlé du * colonialisme " culturel français ${ }^{21}$ et il s'évertue à montrer, dans des livres comme La naissance d'une race, que les Canadiens français forment maintenant une race modifiée, un peuple avec une personnalité distincte. Il affiche également un sentiment d'antagonisme, voire d'hostilité assez ouverte, face au Canada anglais, coupable, dit-il, de soumettre le Québec à un «servage

21 Lionel Groulx, "Notre histoire, L'action française, vol. II, n* 8, août 1918 , p. 343. 
impérialiste 22 . Ainsi, même si le mouvement régionaliste sous l'abbé Groulx reste essentiellement conservateur (basé sur l'histoire, les traditions, etc.), élitiste et fort catholique, donc moralisateur et idéaliste, il aborde néanmoins de front les questions politiques et économiques de l'époque ${ }^{23}$. Le roman L'appel de la race (publié sous le pseudonyme d'Alonié de Lestres), qui crée un si grand scandale en 1922, est remarquable à cet égard; il situe à Ottawa (donc en ville) la lutte pour la survie de la langue française, et aborde des questions tout à fait contemporaines telles que les mariages mixtes (c'est-à-dire entre les deux * races n), la séparation des couples mariés, ou la lutte parlementaire pour les droits linguistiques des Franco-Ontariens. Il s'agit donc d'un régionalisme véritablement "nationaliste" dans le sens où, toutes proportions gardées, il tend vers la formation d'une nation autonome qui constituerait son propre centre culturel et politique. Son nationalisme fort conservateur n'est d'ailleurs pas exempt d'une certaine dose de racisme.

C'est Léo-Paul Desrosiers, disciple de l'abbé Groulx et futur auteur des Engagés du Grand Portage (roman historique fort réussi), qui résumera le mieux les conséquences logiques de cette deuxième forme de régionalisme. Après avoir soutenu très habilement, avec beaucoup d'élégance et d'intelligence, une longue polémique avec Victor Barbeau, qui avait déclaré la guerre au régionalisme littéraire dans $\mathrm{La}$ presse, Desrosiers en arrive à la conclusion que ce qu'il défend et souhaite, en fait, ce n'est pas le régionalisme mais la littérature canadienne-française ${ }^{24}$. Et il aurait pu ajouter: "autonome ".

Ce qui est certain, en même temps, c'est qu'il n'est surtout pas question, pour ces régionalistes, d'une autonomisation de la littérature telle qu'elle est conçue par Pierre Bourdieu ${ }^{25}$. La littérature canadienne-française doit, selon eux, se soumettre avant tout aux exigences idéologiques de la religion et du patriotisme.

22 Ibid. Comme on le voit par ces deux exemples, le discours de Groulx fait parfois étonnamment moderne...

23 La Ligue des Droits du français, dont L'action française est l'organe, prône une conduite autrement plus engagée politiquement et socialement que celle qui était préconisée par Le parler franģais.

24 Le lien problématique entre Desrosiers et le programme de Groulx rappelle celui de Camille Roy avec le régionalisme prôné par Adjutor Rivard.

25 Pierre Bourdieu, :Le marché des biens symboliques *, L'année sociologique, $\mathrm{n}^{\circ} 22,1971$, p. 49-126. 
18

L'autonomie recherchée devait se faire plutôt par rapport à la littérature française officielle, et il faut admettre qu'ils ont remporté une certaine victoire sur ce chapitre.

\section{Vers une institution littéraire canadienne}

Poursuivant les efforts déjà tentés en ce sens par la génération de 1860 (et, même s'ils ne l'auraient peut-être pas admis, par l'École littéraire de Montréal), les divers mouvements régionalistes ont réussi à doter le Québec des bases d'une institution littéraire autonome ${ }^{26}$. Examinons d'abord le rôle joué par les membres de la Société du Parler français: Camille Roy met sur pied la première bistoire de la littérature canadienne-française; lui et Adjutor Rivard, avec d'autres de l'époque ${ }^{27}$, tentent d'établir une véritable critique littéraire, moins partisane qu'auparavant. En prévision du premier Congrès de la langue française au Canada en 1912, la Société institue un premier concours littéraire, et donc des prix littéraires, donnant ainsi un exemple qui sera suivi d'abord par la Société Saint-Jean-Baptiste de Montréal (19161919), puis par le gouvernement, avec la création du prix David. Les séances annuelles de la Société, auxquelles assistait une part considérable de l'élite canadienne-française, tant ecclésiastique qu'intellectuelle, avaient également un grand impact, surtout du point de vue de la légitimisation et de la consécration de leur œuvre. Il ne faudrait pas non plus sous-estimer l'importance de leur action auprès des jeunes, avec tous les Tableaux et Manuels de littérature canadienne-française fournis par Camille Roy, les Prix du Bon Parler français et même les centres d'étude fondés par la Société directement dans les écoles. De ce point de vue-là, cependant, L'action française, sous l'égide de l'abbé Lionel Groulx, se montrera, si possible, encore plus habile. Ses partisans, qui apportent un zèle exemplaire à répandre ce qu'ils appellent fièrement leur "propagande ", s'assureront des entrées privilégiées auprès des écoles, où ils fournissent des prix, des abonnements, etc. Ils fonderont aussi leur propre maison d'édition. Et comment évaluer l'importance du rôle joué par Groulx dans la fondation de

26 Il est essentiel de noter ici que, à l'encontre de Jacques Dubois, je fais une distinction très nette entre l'institution littéraire et l'autonomisation de la littérature telle qu'elle est décrite par Pierre Bourdieu.

27 On peut penser ici aux efforts de Louis Dantin, de Charles ab der Halden ou de Germain Beaulieu, entre autres. 
l'ACJC ainsi que dans l'interprétation et la vulgarisation de l'histoire canadienne-française sous un angle fortement rationaliste?

\section{Scissions}

La prise de position plus nationaliste, et moins régionaliste, de L'action française ne rencontre pas l'approbation de tous. Elle est un facteur important dans la décision des "exotiques" de se regrouper afin de faire opposition au régionalisme, et elle provoque des scissions au sein du camp régionaliste. En effet, les premiers grands défenseurs de cette cause, Adjutor Rivard et Camille Roy, semblent se sentir obligés, face à l'extrémisme de Groulx, de prendre un peu de distance vis-à-vis du régionalisme. Dans son Manuel d'bistoire de la littérature canadienne-française de 1918, Roy tente subtilement de minimiser, voire de -dénationaliser ", son programme de nationalisation esquissé en 1904. Dans une conférence intitulée *Ã propos de proverbes ", prononcée en avril 1920, Adjutor Rivard dénonce certains abus auxquels le régionalisme aurait donné lieu et insiste sur la nécessité pour le Québec de se voir comme une "province intellectuelle de la France" et de ne pas tourner le dos à son héritage culturel et littéraire ${ }^{28}$.

Dans toutes ces discussions, la question de la langue n'est jamais très loin et, en novembre 1920, dans le cadre des :Soirées de l'Action française ", Henri d'Arles ira jusqu'à prononcer un discours en faveur d'une langue canadienne. Pour lui, ce n'est que l'aboutissement logique de la perspective défendue par Groulx : : Or, une race qui possède une aire parfaitement homogène, et toutes les caractéristiques qui font les nations, doit avoir sa langue à elle. La langue est l'expression de la pensée."29 Devant de telles affirmations, Louvigny de Montigny crie au meurtre (en fait, il crie/écrit *Mugwump*!), alors que le français classique à saveur canadienne qu'il propose lui-même, avec Maria Chapdelaine comme modèle, n'est pas si loin, en fin de compte, du *style canadien * préconisé par d'Arles. Pour celui-ci, en effet, il ne s'agit nullement de s'attaquer à la syntaxe ou à la

28 Adjutor Rivard, •À propos de proverbes (Lu à la séance publique de la Société du Parler français le 21 avril 1920):, Le Canada français, vol. IV, $n^{\circ}$ 5, juin 1920 , p. 406 .

29 Henri d'Arles, :Le parler français. Variations sur notre "parlure" ", Le Canada français, vol. VI, n 1, février 1921, p. 55. 
sémantique du français, mais simplement d'encourager les auteurs canadiens à cultiver leur propre style. Un "style canadien" qui s'alimenterait obligatoirement, précise-t-il, au "parler de nos habitants* (p. 62).

Ce qui fait peur à Louvigny de Montigny, en réalité, c'est que cette sortie en faveur d'une langue canadienne porte la marque d'une école de pensée, celle de "l'Action française [quil fournit des "mots d'ordre" à toute notre jeunesse orthodoxe" et qui voudrait couper le Québec du monde extérieur. Voilà ce à quoi il s'oppose catégoriquement: :Il faut croire la dernière génération désapparentée des traditions ancestrales et imprégnée de la prétention de former une nation indépendante, indépendante dans son esprit et dans ses souvenirs; autant que dans ses intérêts matériels. * 30

Louvigny de Montigny n'est pas pour autant opposé au régionalisme. Il s'agit d'un homme qui a non seulement consacré une bonne partie de sa vie à défendre les qualités de Maria Chapdelaine devant un public canadien souvent récalcitrant, mais qui avait déjà en mars 1904, neuf mois avant le discours de Camille Roy, prôné la nécessité du sujet canadien. Seulement, son "sujet canadien " à lui ne se restreignait pas au terroir et incluait la vie citadine, les démêlés électoraux, etc.

Sommes-nous donc devant une nouvelle forme de régionalisme canadien? Pas vraiment, car Louvigny de Montigny n'avait pas parlé de "régionalisme" en 1904, mais de "littérature nationale. En 1921, en tout cas, il semble accepter la définition du régionalisme mise de l'avant par la Société du Parler français tout en percevant cette "école. comme une possibilité littéraire parmi d'autres:

Quelques-uns de nos poètes et de nos prosateurs se sont appliqués à ce genre lune littérature où on infuse à̀ la bonne langue française l'arôme caractéristique du terroir canadien"], ont parfois réussi à produire des ceuvrettes, sinon encore des œuvres, qui portent distinctement l'empreinte canadienne. Ils ont fait ce qu'Henri d'Arles imagine être du * style national ; en réalité, du régionalisme, c'est-à-dire de la littérature française influencée, conditionnée même par le sol où elle a germé. Cette littérature canadienne ne contrarie nullement la langue clas-

30 Louvigny de Montigny, «Mugwump ", La Revre moderne, vol. II, $\mathrm{n}^{\circ}$ 7, 15 mai 1921, p. 13. 
sique, mais s'y agrège et la ravive par son originalité. À telle enseigne que l'Académie accueille avec gratitude les saines productions régionalistes, de chez nous autant que d'ailleurs, et leur décerne les couronnes réservées aux ouvrages qu'elle reconnaît dignes d'enrichir le patrimoine du génie français. (p. 15)

Cette idée que le régionalisme représenterait seulement une possibilité parmi d'autres pour l'écrivain canadien rappelle beaucoup l'attitude adoptée par Rivard lui-même vers la même époque, alors qu'il tente de se distancier du régionalisme prôné par le cénacle de l'abbé Groulx sans perdre pour autant la paternité du mouvement. Il s'agit pourtant d'un revirement par rapport au message premier véhiculé pendant tant d'années au Parler français, et on ne doit donc pas s'étonner de voir un ancien collaborateur de cette revue, comme Henri d'Arles, évoluer dans le sens de L'action française plutôt que de se dédire à moitié comme le fait Rivard.

\section{Claude-Henri Grignon : pour un régionalisme paysan et réaliste}

Claude-Henri Grignon, en revanche, nous semble représenter à lui tout seul une troisième conception du régionalisme. Du moins, tel est le cas à l'époque où il défend une perspective cohérente et logique sur la question, avant que son goût de la polémique à tout prix ne l'ait enfoncé dans tout un réseau d'opinions contradictoires. Tout en se proclamant résolument régionaliste et en prônant sans arrêt la littérature du terroir, par exemple, Grignon se moque impitoyablement de la plupart des écrivains et des critiques qui souscrivent à ce programme et qui :tombe[nt] d'admiration en face d'un poteau de clôture coiffé d'une chaudière à "laitte" "31. En fait, il serait difficile d'imaginer un allië plus dangereux pour le régionalisme canadien *orthodoxe ". Comme le montrera Un bomme et son péché (peut-être le premier roman canadien-français "régionaliste" vraiment réussi, si l'on fait abstraction de Maria Chapdelaine, œuvre d'un *Français de France"), il n'est pas, comme l'étaient les critiques du Parler français, contre le réalisme. Et il n'est pas, comme l'était Groulx, de tendance séparatiste ${ }^{32}$. Fort conscient de son manque d'éducation formelle et de

31 Claude-Henri Grignon, Ombres et clameurs, Montréal, Éditions Albert Lévesque, 1933, p. 168.

32 Son étude sur Juana, mon aimée d'Harry Bernard, dans Ombres et clameurs, est éloquente à ce sujet. 
22

ses origines soi-disant populaires, Grignon est peut-être, d'une certaine façon, un des premiers "régionalistes * (avec Jean Narrache et Alfred DesRochers) à avoir épousé, dans une perspective non élitiste ${ }^{33}$, une vision populiste des Canadiens français. Il est donc régionaliste dans le sens où il se proclame tel, et il est solidaire des paysans ainsi que de tout ce qui a trait au terroir. (C'est lui qui est l'auteur de la phrase, *Notre littérature sera paysanne ou ne sera pas. *) Il semble rester foncièrement catholique ${ }^{34}$ sans que cela obscurcisse son jugement littéraire. D'ailleurs, son respect de la chose littéraire ainsi que son acceptation du réalisme le rapprochent autant, en fin de compte, de ceux qui luttaient pour l'autonomisation de la littérature au Québec (les *exotiques", grosso modo) que de ceux qui travaillaient pour une littérature canadienne-française autonome. Son éloge dithyrambique de Trente arpents, qu'il accueille comme le grand triomphe du programme régionaliste (même si, comme il le rappelle, Panneton avait œuvré plutôt du côté des opposants de ce mouvement), était donc tout à fait logique. C'est l'œuvre qu'il attendait, qu'il avait peut-être lui-même tenté de faire avec Un bomme et son péché. Il ne semble pas se rendre compte que de telles ouvres, littérairement si réussies, dépassent toute catégorisation de ce genre.

\section{Le canadianisme intégral}

Il y avait une quatrième forme de régionalisme au Québec au début du vingtième siècle, et celui-ci s'est fait connaître sous l'expression de "canadianisme intégral . Même si ce mouvement (si l'on peut l'appeler ainsi, tant son action était diffuse et contestée) préfère se distinguer des "écoles " régionaliste et * exotique * dont les discours antagonistes avaient polarisé la scène littéraire québécoise pendant tant d'années, il constitue néanmoins un aboutissement logique des tentatives antérieures en faveur d'une littérature canadienne-française nationale ou autonome.

33 Cette locution adverbiale, qui peut avoir l'air redondante, est en fait essentielle, car si la plupart des régionalistes ont prôné ou produit des œuvres basées sur les a habitants , et "le peuple ", ils l'ont presque toujours fait avec une certaine condescendance. Et on connaît les connotations qui s'attacheront au spetit peuple * dont parle constamment Lionel Groulx.

34 La fin tragique de Séraphin Poudrier peut d'ailleurs être vue, si l'on étudie bien le roman, comme une punition de Dieu. 
Cette nouvelle tendance "régionaliste " qui s'annonce vers 1930 trouve ses principales expressions dans Paragrapbes d'Alfred DesRochers et dans Carquois d'Albert Pelletier. On y trouve exprimé en même temps le désir d'une littérature canadiennefrançaise autonome et de l'autonomisation de la littérature au Québec. Ce serait une littérature non moralisatrice, libérée des anciens tabous et ouverte aux écoles littéraires modernes telles le réalisme ou le symbolisme. Résolument "canadienne" ou nordaméricaine, tout en permettant la liberté dans le choix du sujet ${ }^{35}$, elle se distinguerait de la littérature française non seulement par la langue ${ }^{36}$, mais aussi par son américanité, élément essentiel, dit-on, du Canadien français moderne. Ce mouvement embryonnaire n'est pas "nationaliste" dans le sens où il est apolitique et ne préconise aucunement une "nation * ou une "race " canadienne-française ${ }^{37}$; il participe même de l'école * exotique * dans la mesure où il tend vers une littérature libérée des contraintes idéologiques, donc vers l'autonomisation de la littérature au Québec. Mais il nous semble être un * régionalisme - dans le plein sens du terme, car il prône une littérature distincte de la littérature française, non seulement par son américanité et sa * canadianité - (les Canadiens français étant, disentils, très différents des Français de France) mais aussi par la langue (cf. Mistral). Il constitue en tout cas l'aboutissement logique des différentes tendances autonomistes qui s'étaient manifestées sur la scène littéraire au Québec depuis une trentaine d'années.

Et pourtant, ce mouvement de "canadianisme intégral ", par son extrémisme même, sonnera en quelque sorte le glas de toute possibilité d'un régionalisme dynamique au Québec à cette époque ${ }^{38}$.

35 DesRochers propose par exemple que le désir ou le rêve de l'ailleurs constitue le thème canadien par excellence.

36 Pelletier, qui est celui qui défend le plus cette idée d'une langue canadienne, voit $\dot{A}$ l'ombre de l'Orford comme un pas dans la bonne direction. Grignon, pour une fois, est tout à fait d'accord.

37 DesRochers subira même une rebuffade importante de la part de ses compatriotes, y compris Louis Dantin, lorsqu'il osera s'inscrire en faux contre la thèse défendue par Albert Lévesque dans La nation canadiennefrangaise. Il rejette la notion de la - race canadienne-française. comme un autre genre de racisme hitlérien et opte plutôt pour une distinction basée sur la langue. Serait Canadien (par opposition à Canadian, dit-il) tout Canadien qui parle et fonctionne en français, et non pas tout membre de la race canadienne-française quelle que soit la langue qu'il pratique.

38 Même si $L e$ terroir de Québec continue à défendre jusqu'en 1939 la cause d'une littérature du terroir au service de l'idéologie agriculturiste, 
Son programme, son désir d'une langue canadienne comme celui d'une littérature idéologiquement autonome, tout cela est absolument irrecevable dans l'institution littéraire québécoise maintenant bien installée.

C'est ainsi que, vers 1931, la plupart des anciens pontifes du régionalisme vont soit prendre leurs distances face au mouvement, soit procéder à une redéfinition de leur position antérieure. Camille Roy, dans Regards sur les lettres (1931), constatera les abus auxquels a donné lieu le mouvement régionaliste, comparé maintenant à un - provincialisme étroit ", et se montrera bien content des nuances (toutes rhétoriques) qu'il avait apportées en 1904, dans son célèbre discours sur "la nationalisation de la littérature canadienne ". Adjutor Rivard, lui, n'avait plus à se dédire, ayant déjà pris ses distances dès 1920. Quant à Louis Dantin, qui en 1903-1904 avait malgré tout reproché à Nelligan son manque de sujets canadiens, il attaquera le mouvement du canadianisme intégral avec énergie en prononçant à Sherbrooke même, devant le cercle d'amis littéraires qui s'était formé autour de la personne charismatique d'Alfred DesRochers, une conférence cinglante contre cette idée complètement farfelue d'une langue canadienne distincte de la française. Son ascendant sur DesRochers lui-même aidera d'ailleurs à éloigner celui-ci de la voie où voulait l'engager son ami Albert Pelletier.

Grignon, par contre, semble avoir bien aimé cette idée de "canadianisme intégral ". Je crois qu'il y aurait volontiers adhéré, n'eût été sa fidélité inébranlable au terroir et à une littérature paysanne.

Quant à l'abbé Lionel Groulx, dont le séparatisme politique s'est radouci avec le temps, sa revue, par l'entremise de ses critiques, l'abbé Charbonnier et Harry Bernard, s'était déjà éloignée de son ancienne approche. Dans les années 1920, après la période la plus explosive de la querelle entre régionalistes et "exotiques", L'action française prônait déjà un régionalisme plus "universel *. En fait, il s'agit maintenant d'un "régionalisme" (car on se réclame toujours de ce terme) qui se cherche et qui prêche tantôt l'imitation du mouvement régionaliste français, tantôt l'idée d'un certain réalisme, tantôt une littérature descriptive quasi synonyme d'exotisme. Ses collaborateurs ne dérogent jamais, cependant, à une perspective foncièrement moralisatrice et catholique.

il fera de plus en plus figure de dinosaure et constatera en 1931 qu'il est à toutes fins pratiques seul à défendre cet idéal. 
Restera Le terroir de Québec qui, sous l'égide de Damase Potvin, continue à défendre jusqu'en 1939 la cause d'une littérature du terroir au service de l'idéologie agriculturiste. Autrement dit, il perpétue, mais de façon nettement plus dogmatique, le programme introduit par Le parler français à partir de 1903-1904. En 1931, déjà, certains propos montrent que ses collaborateurs se sentent de plus en plus comme des chevaliers d'une cause sacrée qu'on trahit partout ailleurs. Leur longue fidélité à un régionalisme résolument agriculturiste finira néanmoins par rendre ces deux termes presque synonymes au Québec.

Même si le "canadianisme intégral ", quant à luil, s'est trouvé en quelque sorte mort-né, ceux que DesRochers désigne sous l'épithète de "la génération de l'après-guerre " (et dont il fait luimême partie) prendront peu à peu leur liberté face aux anciens tabous et contribueront donc de façon importante, comme l'ont fait les "exotiques", à l'autonomisation de la littérature au Québec. Il s'agira d'un combat difficile, bien entendu, mené par des écrivains aussi divers que Jean-Charles Harvey, Ubald Paquin, Jovette-Alice Bernier, Éva Sénécal, Simone Routier, Philippe Panneton, et j'en passe. Même les ouvres littéraires de Louis Dantin, publiées souvent par l'entremise de DesRochers, participeront à cette lente conquête de la liberté.

\section{Et depuis ?}

Il faudra cependant attendre les années soixante et soixantedix pour que réapparaissent, sous une nouvelle poussée autonomiste, certains éléments du discours régionaliste du début du siècle. Naîtra alors une littérature si importante et si autonome que Jacques Dubois (qui ne distingue nullement entre littérature régionaliste et littérature régionale) a du mal à la classer avec les autres littératures francophones hors de France dans la catégorie de "littératures régionales" et qu'il est obligé, pour en parler, de rendre synonymes * régional * et "national *39.

Nous voilà revenus au dilemme terminologique déjà évoqué. Par "littératures régionales", sous-catégorie des "littératures minoritaires", Dubois entend celles "qui, subissant les effets du système institutionnel français, fondé sur l'unification et la centralisation, se trouvent

39 Jacques Dubois, L'institution de la littérature. Introduction à une sociologie, Bruxelles, Nathan/Labor, 1978, p. 135. 
26

géographiquement et culturellement coupées des lieux dominants de production-diffusion et éloignées des instances décisives de consécration* (p. 131). Ainsi la littérature québécoise des années soixante et soixante-dix, qui avait d'autres préoccupations que la reconnaissance parisienne ${ }^{40}$, est-elle venue perturber, par sa qualité indiscutable, la perspective française centralisatrice pour laquelle il n'y avait de littérature française valable qu'encouragée et consacrée par Paris. L'institution française s'est d'ailleurs hâtée de reconnaître certaines des œuvres en question, donnant lieu à cette *vogue des productions québécoises " que Dubois réussit cependant à récupérer dans sa théorie comme "l'occasion de contrebalancer les raffinements de l'art ésotérique par le "naturel" d'un exotisme" (p. 136). (On est toujours l'« exotique * de quelqu'un!)

N'empêche que cette littérature, qui - toujours selon Dubois - - intervient directement sur le terrain politique et idéologique, sans connaître encore la médiation institutionnelle "41, remet en question le "centralisme " de l'institution littéraire française (p. 137). Nous dirions même qu'elle aide à préparer le chemin au mouvement de valorisation de la francophonie auquel on assiste depuis lors. Sans exagérer l'importance de ce concept, sans doute beaucoup moindre en France que dans les autres pays francophones, il reste que son succès témoigne d'une nouvelle attitude. De plus, sa seule existence affirme la possibilité que des littératures "nationales" de langue française se trouvent unies par certaines préoccupations communes.

N'avons-nous pas là un autre exemple du nouveau concept de "régionalisme" né en cette seconde moitié du $\mathrm{xx}^{\circ}$ siècle et qui désigne, ssur le plan international, une tendance à affirmer la solidarité et à promouvoir le développement d'un groupe de nations possédant des intérêts communs ${ }^{42}$ ?

L'histoire récente nous oblige donc à réévaluer les connotations négatives qui se sont historiquement attachées au terme "régionaliste " 43 . Si ce concept fait actuellement l'objet d'un intérêt

40 On se rappellera les mouvements d'indépendance et de décolonisation qui primaient à cette époque-là.

41 Il n'y a d'institution littéraire que parisienne, de toute évidence.

42 Georges Vedel, op. cit., p. 794.

43 Ces connotations négatives ne sont pas sans lien, entre autres, avec le fait que quelques écrivains régionalistes éminents de la France sont devenus des collaborateurs pendant la Seconde Guerre mondiale. 
accru, c'est non seulement parce qu'il évoque la tendance internationale déjà citée, mais aussi parce que, dans son sens plus traditionnel, il constitue un antidote à l'uniformisation radicale du monde contemporain et à $* 1$ 'inadaptation des structures politiques et sociales de l'État classique aux formes nouvelles d'une civilisation dite de masse et de consommation *. Voici ce qu'en dit l'Encyclopaedia universalis:

Pour corriger ces systèmes déshumanisés, le régionalisme postule la valorisation de circonscriptions géographiques plus étroites, donc de collectivités humaines plus réduites. On affiche également la volonté de sauvegarder et d'exalter tous les éléments originaux de ce groupe de base, qu'ils soient d'ordre économique, géographique, linguistique ou folklorique. Même $s^{\prime}$ il est vrai que ces dernières références ont quelquefois donné à la tendance régionaliste une coloration "réactionnaire", il est manifeste que la prise en considération de cette catégorie de valeurs en même temps que la volonté déclarée de répondre aux aspirations individuelles sont de nature à séduire; cela est d'autant plus vrai dans le contexte sociologique actuel, qui lamine les personnalités plus qu'il ne les protège. (p. 795)

Ainsi, le monde contemporain "postmoderne " a beau prendre conscience du caractère éminemment subjectif des termes * identité * ou "nation", il s'agit néanmoins de concepts dont l'être humain a de toute évidence besoin pour son équilibre mental. Le méconnaître serait dangereux, car la répression ou la dissolution de ces sources de certitude entrainent dans bien des cas une folie ou individuelle ou collective. Il n'est pas à la portée de tout un chacun de vivre sans appartenance de groupe, en tant que "citoyen du monde., de même que peu de gens semblent capables de fonctionner sans un sentiment (imaginaire ou non) d'identité personnelle. Aussi, la tendance qu'on désigne sous le terme * régionalisme * n'est-elle pas près de disparaître:

Ces divers éléments [cités ci-dessus] de la tendance régionaliste se retrouvent dans tous les domaines où elle s'exprime, sur le plan national aussi bien que sur celui des communautés internationales, confirmant ainsi le fondement universel d'une tendance qui ne peut être tenue pour une théorie passagère ou contingente. (p. 795) 